\title{
Aldehyde Dehydrogenase, a Therapeutic Target in Chordoma: Analysis in 3D Cellular Models
}

\author{
Marie-Anaïs Locquet ${ }^{1}$, Anne-Lise Dechaume ${ }^{1}$, Paul Berchard ${ }^{1}$, Lhorra Abbes ${ }^{1}$, Daniel Pissaloux ${ }^{2,3}$, \\ Franck Tirode ${ }^{3}{ }^{(0)}$, Inès Ramos ${ }^{1}$, Julie Bedoucha ${ }^{1}$, Julie Valantin ${ }^{4,5}$, Marie Karanian ${ }^{2,3}$, Raul Perret ${ }^{6}$, \\ Olivier Gille ${ }^{7}$, Jean-Yves Blay ${ }^{1,8}$ (1) and Aurélie Dutour ${ }^{1, *}$
}

check for

updates

Citation: Locquet, M.-A.; Dechaume, A.-L.; Berchard, P.; Abbes, L.;

Pissaloux, D.; Tirode, F.; Ramos, I.;

Bedoucha, J.; Valantin, J.; Karanian,

M.; et al. Aldehyde Dehydrogenase, a Therapeutic Target in Chordoma: Analysis in 3D Cellular Models. Cells 2021, 10, 399. https://doi.org/ 10.3390/cells10020399

Academic Editor: Harmen J.G. van de Werken

Received: 16 December 2020

Accepted: 8 February 2021

Published: 15 February 2021

Publisher's Note: MDPI stays neutral with regard to jurisdictional claims in published maps and institutional affiliations.

\section{Copyright: (C) 2021 by the authors.} Licensee MDPI, Basel, Switzerland. This article is an open access article distributed under the terms and conditions of the Creative Commons Attribution (CC BY) license (https:// creativecommons.org/licenses/by/ $4.0 /)$.
1 Team Cell Death and Pediatric Cancer, Cancer Initiation and Tumor Cell Identity Department, INSERM1052, CNRS5286, Cancer Research Center of Lyon, F-69008 Lyon, France;

Marieanais.locquet@lyon.unicancer.fr (M.-A.L.); Annelise.dechaume@lyon.unicancer.fr (A.-L.D.);

Paul.berchard@lyon.unicancer.fr (P.B.); Lhorra.abbes@lyon.unicancer.fr (L.A.);

ines.laurentramos@gmail.com (I.R.); julie.bedoucha@gmail.com (J.B.);

Jean-yves.blay@lyon.unicancer.fr (J.-Y.B.)

2 Department of Biopathology, Centre Leon Berard, F-69008 Lyon, France; Daniel.pissaloux@lyon.unicancer.fr

3 Team Genetics, Epigenetics and Biology of Sarcomas, Univ Lyon, Université Claude Bernard Lyon 1, INSERM1052, CNRS5286, Cancer Research Center of Lyon, Centre Leon Berard, F-69008 Lyon, France; Franck.tirode@lyon.unicancer.fr (F.T.); Marie.karanian@lyon.unicancer.fr (M.K.)

4 Research Pathology Platform, Department of Translational Research and Innovation, Centre Leon Berard, F-69008 Lyon, France; Julie.valantin@lyon.unicancer.fr

5 Fondation Synergie Lyon Cancer, F-69008 Lyon, France

6 Department of Biopathology, Institut Bergonié, F-33000 Bordeaux, France; r-perret@bordeaux.unicancer.fr

7 Orthopedic Spinal Surgery Unit 1, Bordeaux University Hospital, F-33000 Bordeaux, France;

Olivier.gilles@chubordeaux.fr

8 Medical Oncology Department, Centre Leon Berard, F-69008 Lyon, France

* Correspondence: Aurelie.dutour@lyon.unicancer.fr

Abstract: Chordomas are rare, slow-growing tumors of the axial skeleton. These tumors are locally aggressive and refractory to conventional therapies. Radical surgery and radiation remain the first-line treatments. Despite these aggressive treatments, chordomas often recur and secondline treatment options are limited. The mechanisms underlying chordoma radioresistance remain unknown, although several radioresistant cancer cells have been shown to respond favorably to aldehyde dehydrogenase (ALDH) inhibition. The study of chordoma has been delayed by small patient cohorts and few available models due to the scarcity of these tumors. We thus created cellular 3D models of chordoma by using low-adherence culture systems. Then, we evaluated their radiosensitivity using colony-forming and spheroid size assays. Finally, we determined whether pharmacologically inhibiting ALDH increased their radiosensitivity. We found that 3D cellular models of chordoma (derived from primary, relapse, and metastatic tumors) reproduce the histological and gene expression features of the disease. The metastatic, relapse, and primary spheroids displayed high, medium, and low radioresistance, respectively. Moreover, inhibiting ALDH decreased the radioresistance in all three models.

Keywords: chordoma; 3D models; hypoxia; radiotherapy; aldehyde dehydrogenase; radioresistance; combination therapy

\section{Introduction}

Chordomas are rare bone tumors of the axial skeleton that localize preferentially in the cranial and the sacral areas. With an incidence of 700 new cases per year, chordomas account for $1-4 \%$ of bone tumors and $20 \%$ of spinal tumors [1]. These tumors, thought to arise from embryonic remnants of the notochord, can affect people at any time through life even if the median age at diagnosis is between 50 and 60 years [2,3]. Conventional chordomas possess some peculiar characteristics. They are slow growing tumors and 
are locally aggressive, invading surrounding structures including the bone and often compressing important structures such as cranial nerves or the spinal cord [4]. They are almost avascular, leading to strong hypoxia, and are composed of large, vacuolated, physaliphorous cells surrounded by an abundant mucoid extracellular matrix [5].

As in the vast majority of bone sarcoma, chordoma patient management consists of surgical resection of the tumor [6]. The goal of resection is to obtain adequate margins, and the quality of surgery is the most important prognostic factor [4]. However, resection with adequate margins is achieved in roughly $50 \%$ of cases, mainly due to the location of the tumor in the vicinity of important structures [4]. In the case of inadequate margins, neoadjuvant therapy is combined with surgery. Among all neoadjuvant therapies, highdose conventional radiotherapy remains the only option, with doses superior to 70 Gy $[7,8]$. Despite its low accessibility, proton therapy can be useful because it allows the delivery of a more important dose to a tumor without exposing surrounding tissues to excessive toxicity [9]. Despite this aggressive treatment, the 5-year relapse-free survival rate is only $50 \%$ [8]. Treatment options are limited at relapse due to the invasiveness and toxicity of the first-line treatment. Hence, there is a need for therapies addressing chordoma specificities. To achieve this, a better understanding of chordoma biology and of the mechanisms underlying chordoma radioresistance is of utmost importance. Decreasing the radioresistance of these tumors could decrease the toxicity triggered by radiations, could improve patient care, and could improve tumor control. The scarcity of these tumors makes large-scale molecular studies challenging, and only a few molecular alterations have so far been described [10]. Although the causes of radioresistance in chordoma are still largely unknown, several factors may be involved. Indeed, hallmarks of these tumors are their slow growth and their low number of mitotic cells, which are the principal targets of radiotherapy [11]. Moreover, the low level of vascularization leads to strong hypoxia, which represents a major predictive factor of unfavorable tumor response to radiotherapy $[12,13]$.

To improve our understanding of the mechanisms underlying chordoma radioresistance, we need to reconstitute the peculiar characteristics of these tumors. Because of their scarcity, only a few models of these tumors are available: 20 cell lines and 11 patientderived xenograft models. Moreover, cells grown in 2D do not reflect some of the major characteristics of chordoma such as hypoxia, extracellular matrix production, and disease progression [14]. To address these issues, we hypothesized that creating cellular 3D models of chordoma could improve our understanding of the mechanisms of radioresistance. Thus, the aims of our study were (i) to create cellular 3D models of chordoma that reproduce their histological and morphological features, (ii) to evaluate their resistance to radiations, and (iii) to determine whether a radiosensitizing approach could be applicable. In this regard, we tested the effectiveness of targeting aldehyde dehydrogenases (ALDHs) 1 and 3. Indeed, ALDHs are a family of enzymes involved in cell detoxification [15]. They oxidize a wide range of endogenous and exogenous aldehydes and protect living organisms from oxidative stress [6]. A high ALDH activity has already been correlated with resistance to treatment in multiple solid tumors. For instance, ALDH-positive breast cancer cells are radioresistant in vitro $[16,17]$. ALDH1-positive cells exhibit a radioresistant phenotype enhanced with hypoxia in cervical cancer [18]. ALDH activity promotes radioresistance in prostate cancer progenitor cells [19] and is indicative of head and neck squamous cell carcinoma (HNSCC) radioresistant cells [20]. This is also the case for bone sarcoma, in which a high ALDH activity is associated with chemotherapy-resistant Ewing sarcoma cells [21]. A population of cells with a high ALDH activity is known to be present in both chordoma patients [22] and cell lines U-CH1 [23], U-CH2, UM-Chor1 [24], and JHC7 [25]. Moreover, the inhibition of ALDH decreases the radioresistance of breast, prostate, and cervical cancer cells [26-28]. Collectively, these findings suggest a central role for ALDH in cellular resistance to radiations. However, the involvement of ALDH in radioresistance has never been studied in chordoma. 
Hence, we created cellular 3D models of chordoma by using ultra-low adherence culture systems. We evaluated their radiosensitivity and determined whether pharmacologically inhibiting ALDH1/3 decreased the radioresistance of our models.

\section{Materials and Methods}

2.1. Cell Lines

2.1.1. Cultures

Conventional chordoma cell lines U-CH12, U-CH1, and CH22 established from primary, relapse, and metastatic chordoma, respectively, were kindly provided by the Chordoma Foundation (Durham, NC, USA). The U-CH1 and U-CH12 cell lines were maintained in 2D cultures on rat tail collagen I-coated flasks (A1048301, Thermofisher, Illkirch, France) in Iscove Modified Dulbecco Media (IMDM):Roswell Park Memorial Medium (RPMI) 4:1 medium (Life technologies, Carlsbad, CA, USA) supplemented with insulin, transferrin, and selenium $1 \%$ (ITS, Life technologies) for U-CH12. The $\mathrm{CH} 22$ cell line was maintained on non-coated flasks in RPMI medium. All media were supplemented with $10 \%$ fetal bovine serum (FBS) and $1 \%$ penicillin streptomycin (PS).

\subsubsection{Cell Culture Conditions and Treatments}

Chordoma spheroids were generated by seeding 2500 cells from either cell lines in 96 ultra-low attachment (ULA) plates (Corning ${ }^{\circledR} \operatorname{Costar}^{\circledR}$, Brumath, France) in the same media as used for 2D cultures. Once seeded, spheroid formation was examined twice a week under an optical inverted microscope (Zeiss, Göttingen, Germany). Spheroids were allowed to form for 7 days before being treated either with radiation or an ALDH inhibitor. A 2 Gy radiation dose was applied to spheroids (dose rate of $6 \mathrm{~Gy} \mathrm{~min}^{-1}$ ) with a $6 \mathrm{MeV}$ X-ray clinical irradiator (SL 15 Phillips). The irreversible inhibitor of ALDH1 and ALDH3, DIMATE (Advanced BioDeisgn, Saint Priest, France), was added to spheroids for 24 to $72 \mathrm{~h}$ (concentrations range: 0.1 to $25 \mu \mathrm{M}$ ).

\subsection{Proliferation Assay}

Spheroid proliferation was assessed at days $4,7,10,15$, and 21 by CellTiter-Glo ${ }^{\circledR}$ luminescent cell viability assay (CTG) (Promega, Charbonnières-les-Bains, France). Each spheroid was washed with PBS1X and incubated with $60 \mu \mathrm{L}$ of CTG in a medium devoid of serum (ratio 1:1). Luminescence was detected after $25 \mathrm{~min}$ of incubation on a TECAN Infinite 500 reader (TECAN, Männendorf, Switzerland) for $500 \mathrm{~ms} /$ well. After $48 \mathrm{~h}$ of treatment, spheroid proliferation in response to increasing concentrations of DIMATE ( 0.1 to $25 \mu \mathrm{M}$ ) was measured by Cell Titer Glo to determine DIMATE IC50.

\subsection{Real-Time Monitoring}

Spheroid proliferation was also determined using real-time monitoring with an IncuCyte Live Cell Analysis System (Incucyte ZOOM, Essen Bioscience, West Wickham Kent $\mathrm{BR} 4 \mathrm{OPH}, \mathrm{UK})$. Spheroid diameters were measured using the IncuCyte software. Spheroid cell death induced by radiation and/or DIMATE treatment was monitored in real-time using the IncuCyte. Irradiated and/or DIMATE-treated spheroids were incubated with $50 \mu \mathrm{L}$ of Cytotox Green reagent (4633, Essen Bioscience) in phenol red-free medium according to the manufacturer's instructions and placed under the $20 \times$ objective lens of the IncuCyte Live Cell Analysis System over a period of $48 \mathrm{~h}$. The evolution of Cytotox Green fluorescence intensity was then analyzed with the IncuCyte software.

\subsection{Immunostaining}

Spheroids were harvested at day 7, 10, 15, and 21 after seeding; fixed in eosin 1:100 and PFA $4 \%\left(2 \mathrm{~h}, 4{ }^{\circ} \mathrm{C}\right)$; embedded in paraffin before cutting into $5 \mu \mathrm{m}$ sections; and stained. HPS and Ki67 staining were performed on an automated Ventana Discovery XT staining system (Ventana Medical Systems, Innovation PARk Drive, Roche, Tucson, AZ 85,755, USA). The Ki67 index was assessed semi-quantitatively. For hypoxia staining, the 
sections were incubated with pimonidazole ( $100 \mu \mathrm{g} / \mathrm{mL}$ for $1 \mathrm{~h}$ at room temperature (RT) (Hypoxyprobe, MA, USA) before fixation.

After the deparaffination, rehydration, and antigen retrieval steps (citrate buffer, $\mathrm{pH}$ 6.0, $95^{\circ} \mathrm{C}, 15 \mathrm{~min}$ or Tris EDTA, $95^{\circ} \mathrm{C}, 15 \mathrm{~min}$ ), the slides were incubated overnight $\left(4{ }^{\circ} \mathrm{C}\right)$ with the following primary antibodies: anti-brachyury(1:500), anti CD24 (1:100), 4-hydroxynonenal (Abcam, Cambridge, UK), anti-cytokeratin AE1 / AE3 (1:100), anti EMA (1:250) (Dako, Agilent, Santa Clara, CA, USA), anti $\gamma \mathrm{H} 2 \mathrm{AX}$ (1:500) (Cell signaling technology, Leyde, The Netherland), anti HIF-1 $\alpha$ (1:100), anti-ALDH3A2 (1:100) (Proteintech, Manchester, UK), or anti-pimonidazole (Hypoxyprobe). Endogenous peroxidases were inactivated by incubating the slides in oxygen peroxide $(0.3 \%, 15 \mathrm{~min}, \mathrm{RT})$. The primary antibodies were detected with biotinylated goat anti-rabbit or goat anti-mouse secondary antibody (VectorLab, Burlingame, United States, 1:100, $1 \mathrm{~h}$, RT) followed by an avidinbiotin complex and DAB peroxidase (SK-4100, Vector Lab; dilution 1:300, $30 \mathrm{~min}, \mathrm{RT}$ ). The sections were counterstained in hematoxylin (Vector Lab, H3401) and mounted in Vectamount medium (H-5000). For aldehyde staining, the sections were incubated with secondary antibody (goat anti-mouse Alexa fluor 488, Abcam, 150113) and DAPI (1:1000, Abcam, 228549) for $1 \mathrm{~h}$ at RT. The sections were then mounted on slides using Fluoromount (Sigma, Merck, Darmstadt, Germany). For extracellular matrix staining, the slides were incubated with alcian blue (Sigma, B8438) and sirius red (Sigma, 2610) for $10 \mathrm{~min}$ at RT. The sections were washed and counterstained with fast red or hematoxylin, respectively. All slides were examined under a Zeiss Axioimager M2 microscope (SIP 60549, Zeiss, Göttingen, Germany), and quantifications were made using the ImageJ software.

\subsection{Electron Microscopy}

Spheroids were fixed in buffered aldehyde ( $4 \%$ formaldehyde, $2 \%$ glutaraldehyde, $1 \mathrm{mM} \mathrm{MgCl} 2$, and $1 \mathrm{mM} \mathrm{CaCl}_{2}$ in $100 \mathrm{mM} \mathrm{Ca-cacodylate,} \mathrm{pH}$ 7.2), post-fixed in aqueous $1 \%$ osmium tetroxide, and then dehydrated in graded steps of ethanol. The spheroids were impregnated in Epon A (50\%), Epon B (50\%), and DMP30 (1.7\%) and were included for polymerization $\left(60^{\circ} \mathrm{C}, 72 \mathrm{~h}\right)$. The spheroids were cut in ultrathin $70 \mathrm{~nm}$ sections before being contrast-stained with lead-citrate and uranyl acetate and imaged with a Jeol 1400JEM (Tokyo, Japan) transmission electron microscope at the CIQLE (Centre d'Imagerie Quantitative Lyon Est).

\subsection{Self-Renewal}

Self-renewal of the chordoma spheroids was assessed by colony formation assays and spheroid size assays. After irradiation and/or DIMATE treatment, the spheroids were maintained $24 \mathrm{~h}$ in culture before being dissociated using the TryPLE reagent $(5 \mathrm{~min}$, $37^{\circ} \mathrm{C}$, Thermofisher, Illkirch, France) followed by gentle pipetting to obtain single cell suspensions.

Two thousand or five thousand cells were seeded onto 6-well plates and were allowed to form colonies for 5 doubling times ( 2 weeks for $\mathrm{CH} 22,5$ weeks for $\mathrm{U}-\mathrm{CH} 12$, and 7 weeks for U-CH1). The colonies were counterstained with a solution of ethanol $75 \%$ crystal violet (Sigma, V5265) and counted using the ImageJ software. To perform a spheroid size assay, 200 single cells were seeded in ULA 96-well plates and were allowed to form spheres for 15 days. All spheres $(>50 \mu \mathrm{m})$ in a well were manually imaged, counted, and measured (diameter) using a Zeiss Axio Observer Z1 inverted microscope (Zeiss, Göttingen, Germany).

\subsection{Aldefluor Assay}

ALDH enzymatic activity was measured using the Aldefluor kit (Stem Cell Technologies). The cells suspended in Aldefluor assay buffer were incubated with an ALDH enzyme substrate for $40 \mathrm{~min}$ at $37^{\circ} \mathrm{C}$. As a control for baseline fluorescence, the cells were also treated with ALDH inhibitor diethylaminobenzaldehyde (DEAB). The cells were washed and stained with viability dye (Miltenyi Biotec, Paris, France) at 1:1000. Fluorescence 
was detected using a BD Bioscience LSR Fortessa flow cytometer and analyzed using the FACSDiva software (BD Biosciences, San Jose, CA, USA).

\subsection{RNA Sequencing}

RNA sequencing was performed for both spheroids after 7 days of culture and patient samples. Total RNA was extracted from macro-dissected formalin-fixed paraffin-embedded tumor sections using the FormaPure RNA kit (Beckman Coulter \#C19158, Brea, CA, USA). The RNase-free DNase set (Qiagen \#AM2222, Courtaboeuf, France) was used to remove DNA. RNA quantification was assessed using NanoDrop 2000 (Thermo Fisher Scientific, Waltham, MA, USA) measurement and RNA quality using theDV200 value (the proportion of RNA fragments larger than $200 \mathrm{nt}$ ) assessed by a TapeStation with Hs RNA ScreenTape (Agilent, Santa Clara, CA, USA). Samples with sufficient RNA quantity $(>0.5 \mu \mathrm{g})$ and quality (DV200 > 30\%) were further analyzed by RNA sequencing. One-hundred nanograms of total RNA were used to prepare libraries with the TruSeq RNA Exome (Illumina \#20020183, San Diego, CA, USA). Twelve libraries were pooled at a concentration of $4 \mathrm{nM}$ each, together with $1 \%$ PhiX. Sequencing was performed (paired end, $2 \times 75$ cycles) using NextSeq $500 / 550$ High Output V2 kit on a NextSeq 500 machine (Illumina).

Alignments were performed using STAR on the GRCh38 version of the human reference genome. The number of duplicate reads were assessed using PICARD tools. No sample was discarded from the analysis (number of unique reads above 10 million). The expression values were extracted using Kallisto version 0.42.5 tool17 with GENECODE release 23-genome annotation based on the GRCh38 genome reference. The Kallisto TPM expression values were transformed in $\log 2(\mathrm{TPM}+2)$, and all samples were normalized together using the quantile method from the $\mathrm{R}$ limma package within $\mathrm{R}$ (version 3.1.1) environment. tSNE analysis was performed to visualize the distance between chordoma spheroids, patient samples, and a series of 1450 sarcoma samples with at least 144 molecular subtypes, as previously published [29]. A classic differential gene expression analysis was conducted between chordoma samples and spheroids.

\subsection{Patients Samples and Ethics}

The study was approved by the Clinical Research Ethics Committee of the Centre Leon Berard, ethical approval number 2018-014, approval date 3 October 2018. A total of 13 patients with conventional chordoma were included in the study. The clinical informations are presented in Table 1.

Table 1. Patients clinical informations

\begin{tabular}{cccccc}
\hline Age & Sex & Tumor Location & & Treatment & $\begin{array}{c}\text { Local Relapse or } \\
\text { Metastasis }\end{array}$ \\
\hline 84 & F & Sacrum & Primary & Surgery & No \\
77 & F & Sacrum & Primary & Surgery & No \\
70 & M & Sacrum & Primary & Surgery & Local relapse \\
59 & F & Sacrum & Primary & Surgery & Local relapse \\
67 & M & Sacrum & Primary & No surgery & No \\
70 & M & Sacrum & Primary & No surgery & No \\
56 & M & Sacrum & Primary & Surgery & No \\
36 & M & Sacrum & Primary & RT & No \\
66 & M & Sacrum & Primary & Surgery & No \\
72 & F & Sacrum & Primary & Surgery & No \\
57 & M & Sacrum & Primary & Surgery & No \\
40 & M & Crane & Metastasis & No surgery & No \\
\hline
\end{tabular}

\subsection{Statistical Analysis}

GraphPad Prism (GraphPad Software, Inc., La Jolla, CA, USA) v8.0 was used for the graphic representation and statistical analysis of the data. The comparison between groups in all experiments was determined using analysis of variance in GraphPad v8.0. Tukey's 
test or Sidak's test were used in the post analysis for one-sided and two-sided statistical analyses, respectively. The differences were considered significant if the $p$-value was $\leq 0.05$.

\section{Results}

\subsection{Chordoma Spheroids Recapitulate the Main Histological and Morphological Features of the Disease}

Because the few available cellular models of chordoma do not fully recapitulate the structures of these tumors, we generated 3D cellular models of chordoma originating from three cell lines representative of the three different stages of the disease: (i) primary tumor U-CH12, (ii) locally relapsed tumor U-CH1, and (iii) metastatic tumor $\mathrm{CH} 22$. We first investigated whether these spheroids faithfully reproduced the most prominent histological and morphological features of the disease.

At the histological level, all three spheroid models expressed the chordomas markers Brachyury, CD24, cytokeratins, and EMA (Figure 1a). While EMA was strongly expressed by the three chordoma spheroid models, Brachyury and CD24 were highly expressed by the $\mathrm{CH} 22$ and U-CH1 spheroids and the U-CH12 spheroids displayed a low expression level. Finally, the $\mathrm{CH} 22$ spheroids alone presented a high expression of cytokeratins (Figure 1a).

Morphologically, consistent with the physaliphorous phenotype of chordoma, spheroid cytoplasm included large vacuoles. Their cytoplasm also held glycogen granules, small aggregates of tonofilaments, and rough endoplasmic reticulum around the mitochondria (Figure 1b). All spheroids presented prominent surface filopodia. At the cellular level, $\mathrm{CH} 22$ spheroids were characterized by very cohesive cells $\mathrm{CH} 22$, joined by tight junctions, whereas U-CH1 and U-CH12 spheroids were less cohesive, only displaying zones of contact between cells (Figure 1b).

Hence, the 3D spheroid models generated recapitulated the main histological and morphological features of chordoma.

Finally, to confirm the validity of our models, we performed RNA sequencing on a cohort of 13 patients and on our spheroids and compared their repertoire of expression to 1450 sarcoma samples, forming at least 144 molecular subtypes. Visualization by tSNE clearly showed that patient samples clustered close to our spheroid samples (Figure S1A). Moreover, a differential gene expression analysis was conducted, highlighting the close relationship between chordoma samples and spheroids (Figure S1B and Tables S1 and S2). These results strongly reinforce the validity of our models.

\subsection{Chordoma Spheroids Recapitulate the Radioresistant Environment of the Disease}

Other chordoma characteristics are the presence of a mucoid extracellular matrix, a strong hypoxia, and a slow progression, at least in their localized form. We then investigated whether our chordoma spheroids present these features.

The presence and composition of ECM in chordoma spheroids was analyzed by sirius red and alcian blue staining, enabling us to visualize collagen and proteoglycans, two major ECM components. Two spheroids U-CH12 and U-CH1 produced their own ECM (Figure 2a). $\mathrm{U}-\mathrm{CH} 12 \mathrm{ECM}$ was rich in collagen fibers, as shown by a more intense sirius red staining, while both U-CH1 and U-CH12 spheroid ECMs contained abundant proteoglycans, as evidenced by alcian blue staining (Figure 2a). 

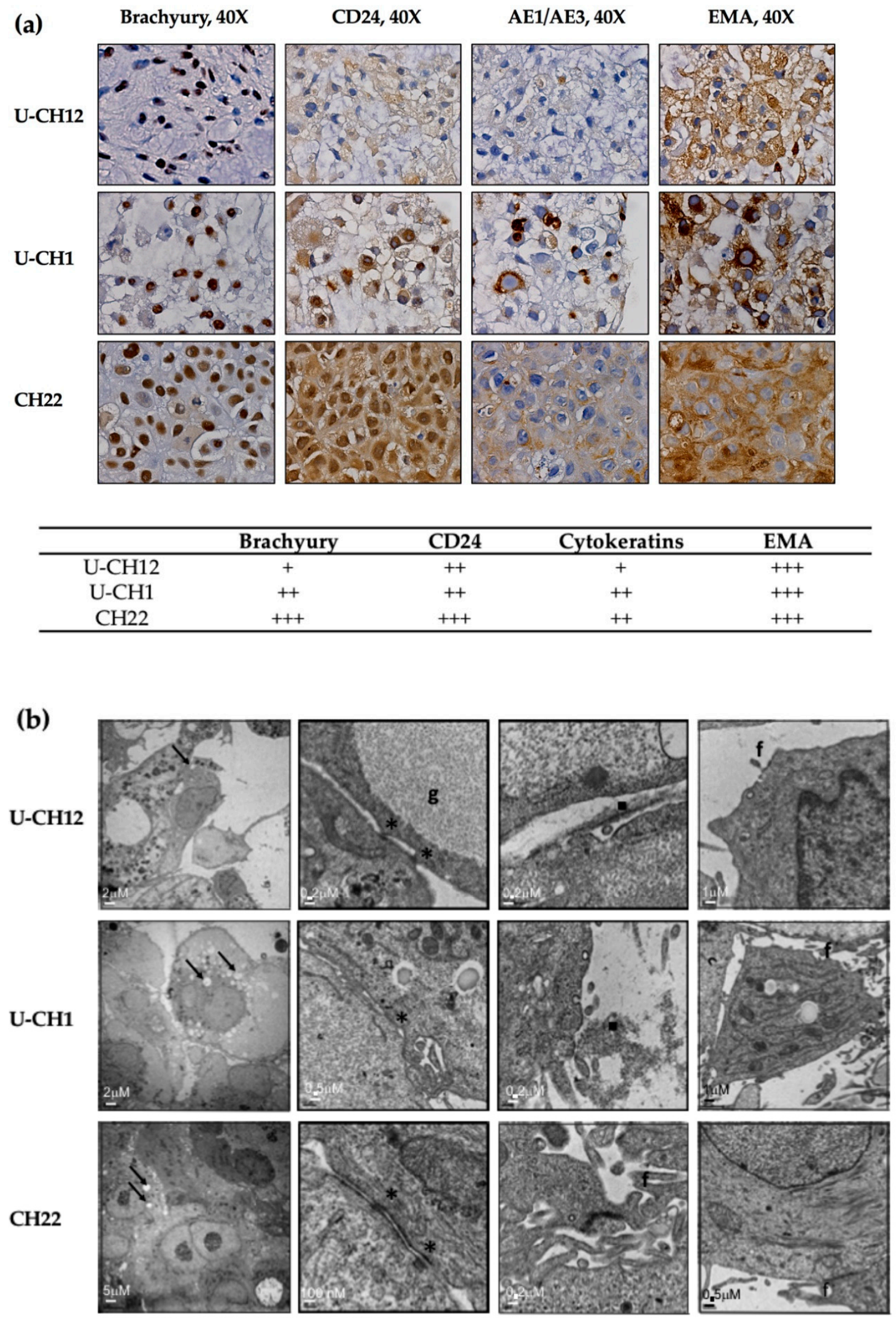

arrow=vacuoles, stars=junctions, $g=g l y c o g e n$, square= extracellular matrix, f=filopodia

Figure 1. Chordoma spheroids recapitulate the main histological and morphological features of the disease: at 7 days of culture, (a) chordoma markers brachyury, CD24, cytokeratins AE1/AE3, and EMA (40× objective) and their quantification were immunostained and (b) spheroid morphology was imaged by an electron microscopy. The arrows represent vacuoles, the stars represent junctions, the squares represent the extracellular matrix, $\mathrm{f}$ is the filopodia, and $\mathrm{g}$ is the glycogen granules. 
(a)

Alcian Blue, 20X Sirius Red, 20X

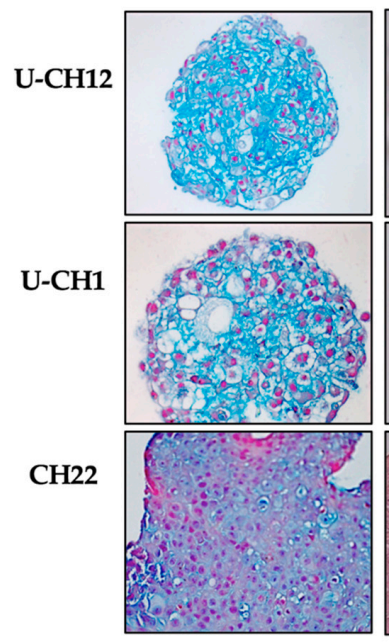

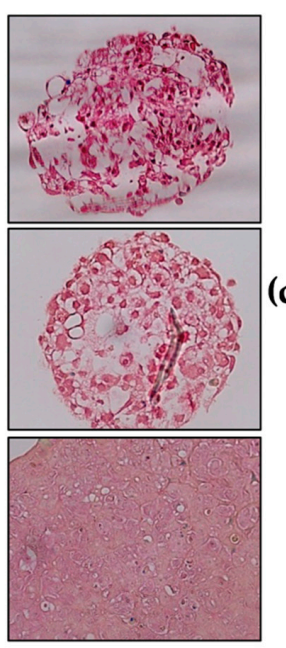

(b)

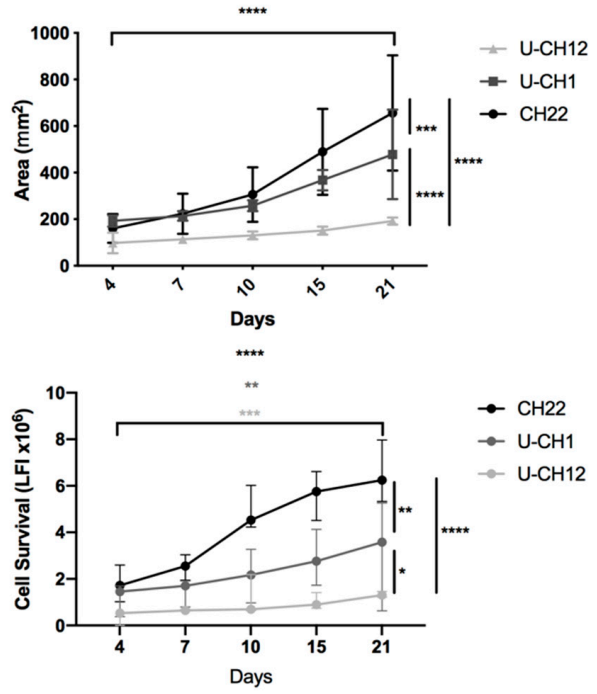

(d)

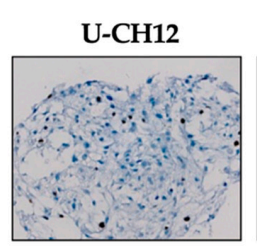

(e)

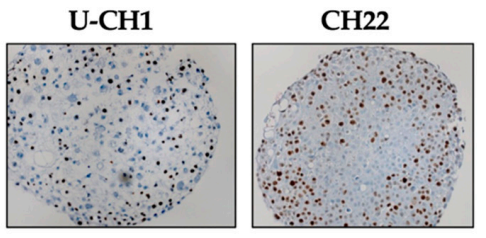

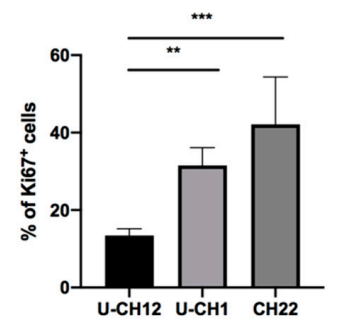

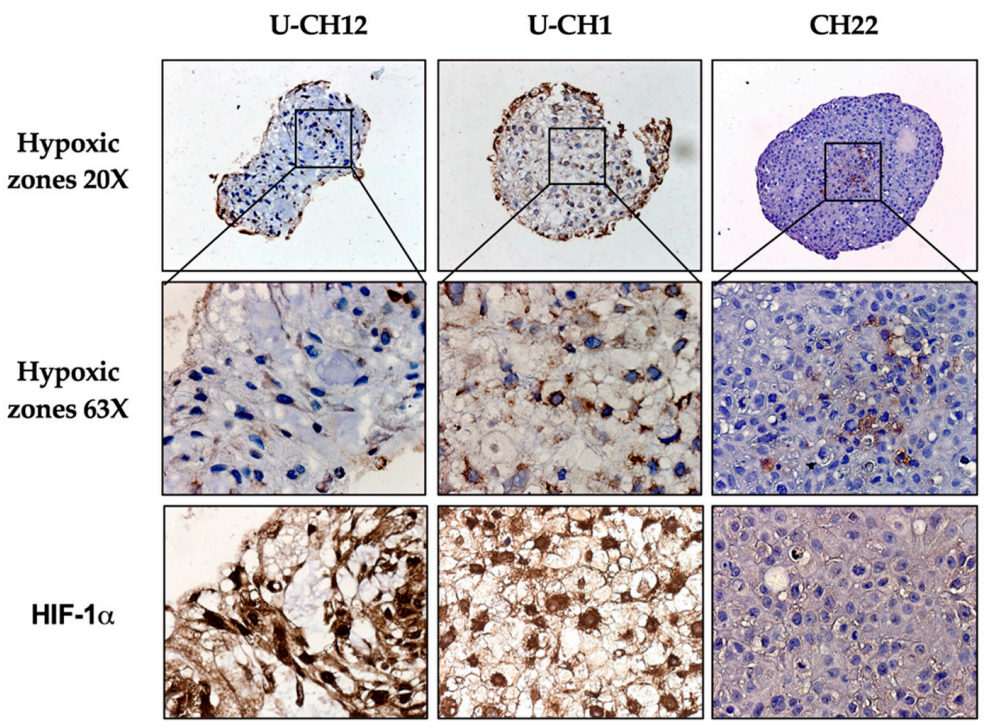

Figure 2. Radioresistant chordoma spheroid environment: at 7 days of culture, (a) extracellular matrix components were immunostained, including proteoglycans with alcian blue and collagen with sirius red ( $20 \times$ objective). (b) The graph represents the median and confidence interval at $95 \%$ of the measure of the area of spheroids using live imaging from day 0 to day 21 after seeding for each spheroid. (c) The graph represents the median and confidence interval at $95 \%$ of the luminescence fluorescence intensity (LFI) correlated with cell survival from day 0 to day 21 after seeding for each spheroid. (d) The number of proliferative Ki67+ cells was evaluated, and the median and confidence intervals at $95 \%$ for each spheroid were quantified. (e) The hypoxic zones within spheroids were mapped by pimonidazole and HIF- $1 \alpha$ staining. Each experiment was conducted in triplicate and repeated three times for spheroid proliferation assessment or twice for extracellular matrix and hypoxia-relative staining. For a comparison of the areas and cell survival, statistical analysis included 2-way ANOVA with time comparison, presented at the bottom of each graph, and cell line comparisons, presented on the right-hand side of the graphs. The comparison of the number of Ki67+ cells between spheroids was determined using a one-way ANOVA analysis. Significant $p$-values are indicated as follows: $p<0.05^{*}, p<0.01^{* *}, p<0.001^{* *}$, and $p<0.0001^{* * * *}$. 
Next, we evaluated spheroid growth by measuring their area over a period of 21 days through live imaging (Figure 2b). CH22 spheroids reached a size of $180 \mathrm{~mm}^{2}$ on day 4 to $740 \mathrm{~mm}^{2}$ on day $21(p<0.0001)$. U-CH1 spheroids were less proliferative $(p<0.001)$ and grew from $190 \mathrm{~mm}^{2}$ on day 4 to $530 \mathrm{~mm}^{2}$ on day $21(p<0.0001)$. The slowest proliferating spheroids were U-CH12 $(p<0.0001)$, growing from $100 \mathrm{~mm}^{2}$ on day 4 to $180 \mathrm{~mm}^{2}$ on day $21(p<0.0001)$. This finding was confirmed via a cell survival assay. $\mathrm{CH} 22, \mathrm{U}-\mathrm{CH} 1$, and U-CH12 showed an increased number of live cells during the 21 days $(p<0.0001$, $p<0.01$, and $p<0.001$, respectively) with three different kinetics. Indeed, $\mathrm{CH} 22$ was the most proliferative (from 1.5 luminescence fluorescence intensity (LFI) on day 4 to $6.25 \mathrm{LFI}$ on day 21) and reached a peak of proliferation between day 7 and day 10. U-CH1 were less proliferative than $\mathrm{CH} 22$ and proliferated uniformly (1.4 to $3.5 \mathrm{LFI}$ ). U-CH12 were the least proliferative spheroids ( 0.52 to $1.3 \mathrm{LFI}$ ) and showed decreased proliferation after day 15 (Figure 2c). Finally, Ki67+ quantification revealed $13.4 \%, 31.5 \%$, and $42.1 \%$ of proliferative U-CH12, U-CH1, and CH22 cells, respectively (Figure 2d).

These patterns of proliferation are representative of the slow growth and progression of chordomas.

Since hypoxia is both a major characteristic of chordomas and a cause of radiotherapy failure, we mapped the hypoxic regions within spheroids using pimonidazole and HIF- $1 \alpha$ staining (Figure 2e). These hypoxic regions were localized at the center of all spheroids and exhibited a nuclear HIF- $1 \alpha$ staining (Figure 2f). Hence, we succeeded in reproducing the hypoxic status of chordoma tumors in our spheroids.

Altogether, these results indicate that our cellular models recapitulate the production of ECM, the slow growth, and the hypoxic status of chordoma, thus mimicking a radioresistant environment.

\subsection{Chordoma Spheroids Exhibit Different Levels of Radioresistance}

As a control, we evaluated radiation-induced DNA damage using yH2AX-stained foci as an indicator of double-stranded breaks after radiation. Thirty minutes after being subjected to $2 \mathrm{~Gy}$ of X-rays, yH2AX foci were present in each spheroid, confirming the efficacy of radiation treatment (Figure 3a). Next, we examined the therapeutic effect of radiotherapy on chordoma spheroid self-renewal and proliferation. A dose of 2 Gy of $\mathrm{X}$-rays resulted in a decrease in the colony forming ability of $\mathrm{CH} 22$ compared to that of untreated spheroids (UT: 132 colonies; 2 Gy: 40 colonies), with only $30 \%$ of colonies remaining after radiation $(p<0.001)$ (Figure $3 b, c)$. In contrast, 2 Gy of X-rays slightly affected U-CH1 and U-CH12, with no significant difference in the number of colonies formed after treatment (UT: 40; 2 Gy: 24; UT: 215; and 2 Gy: 197, respectively) (Figure 3b,c). We then evaluated the sphere forming ability of spheroids after radiation to verify the impact on our 3D model (Figure 3d). After two weeks, the spheres newly formed from irradiated $\mathrm{CH} 22$ spheroids $\left(597,609 \mu^{2}\right)$ had a smaller area than the spheres formed from untreated $\mathrm{CH} 22$ spheroids $\left(2.10^{6} \mu \mathrm{m}^{2} ; p<0.01\right)$ (Figure 3d,e). However, the area of spheres formed from 2 Gy-treated spheroids from both U-CH1 and U-CH12 $\left(224,048 \mu \mathrm{m}^{2}\right.$; $300,000 \mu \mathrm{m}^{2}$ ) displayed no significant difference compared to the area of spheres formed from untreated U-CH1 and U-CH12 spheroids $\left(198,444 \mu \mathrm{m}^{2} ; 286,742 \mu \mathrm{m}^{2}\right.$ ) (Figure $3 \mathrm{~d}, \mathrm{e}$ ).

Hence, our 3 spheroids show 3 different levels of radioresistance, with U-CH12 being the most radioresistant and $\mathrm{CH} 22$ being the least. 
(a) H2AX foci, 100X

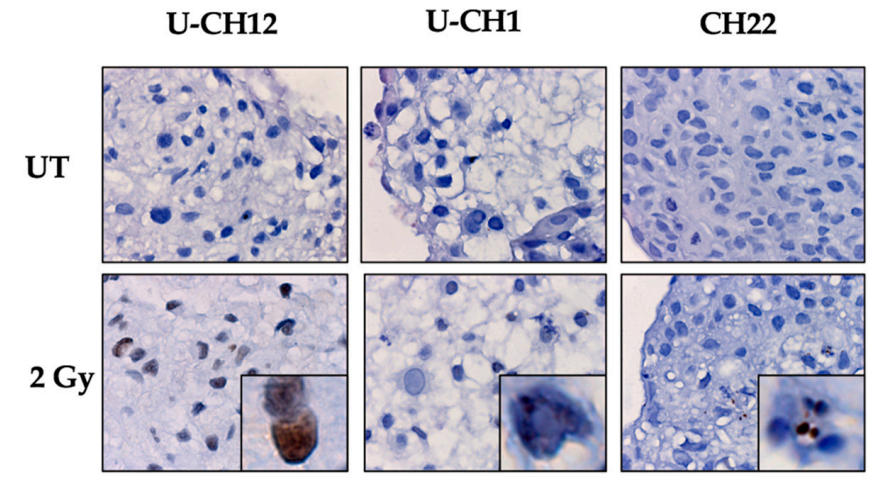

(b)

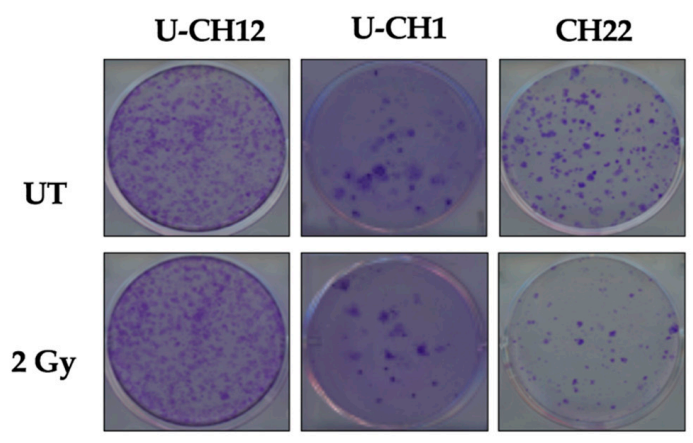

(d)

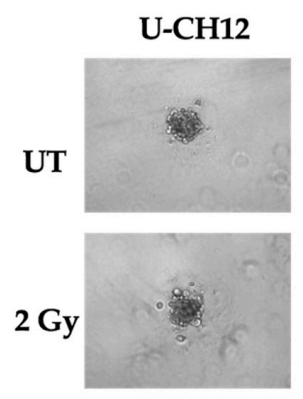

U-CH1
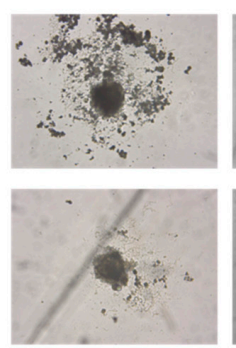

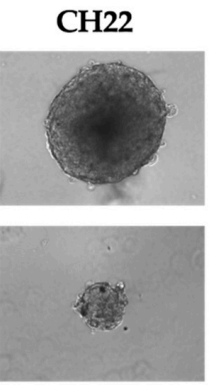

(c)

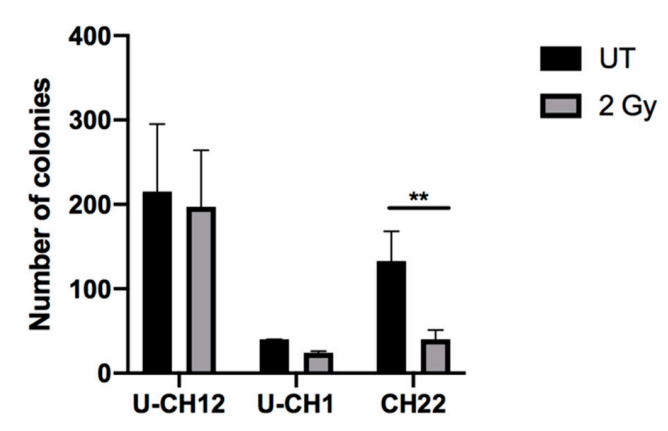

(e)

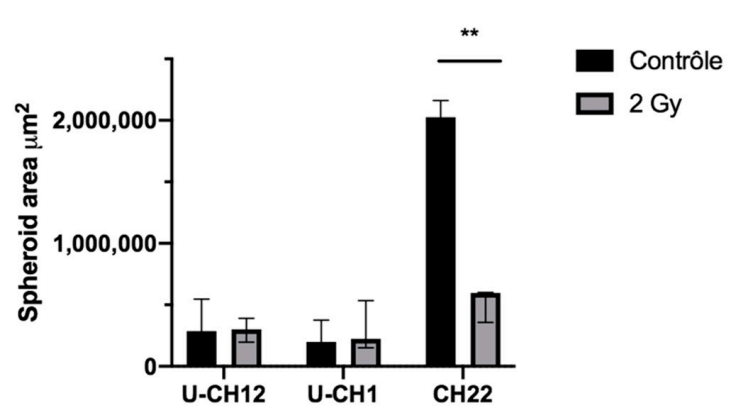

Figure 3. Chordomas exhibit three different levels of radioresistance: (a) DNA double-stranded breaks induced by radiation and quantified using $\gamma \mathrm{H} 2 \mathrm{AX}$ foci staining, (b) images and (c) a graph representative of the number of colonies formed in untreated conditions or after $2 \mathrm{~Gy}$ of X-rays, and (d) images and (e) a graph representative of the spheroid size in untreated conditions or after $2 \mathrm{~Gy}$ of $\mathrm{X}$-rays. Each experiment was repeated three times in triplicate. Comparisons between untreated and radiation-treated groups were analyzed with a two-way ANOVA. Significant $p$-values are indicated as follows: $p<0.01^{* *}$.

\subsection{Aldehyde Dehydrogenase Activity Is a Potential Target in Chordoma}

Aldehyde dehydrogenases are a family of detoxifying enzymes involved in radioresistance of multiple solid tumors. We initially determined whether ALDHs could be potential targets in chordoma by investigating their gene expression using RNA sequencing. A cohort of 13 patients with sacral chordoma, and both $\mathrm{U}-\mathrm{CH} 1$ and $\mathrm{CH} 22$ spheroids were analyzed. We observed that the members of the ALDH3 and ALDH1 families were the most expressed at the transcriptomic level in all samples. Indeed, patient samples and spheroids presented high expression values of ALDH3B1, ALDH3A2, and ALDH1A2 (9, 8, and 7, respectively) (Figure 4a). 
(a)
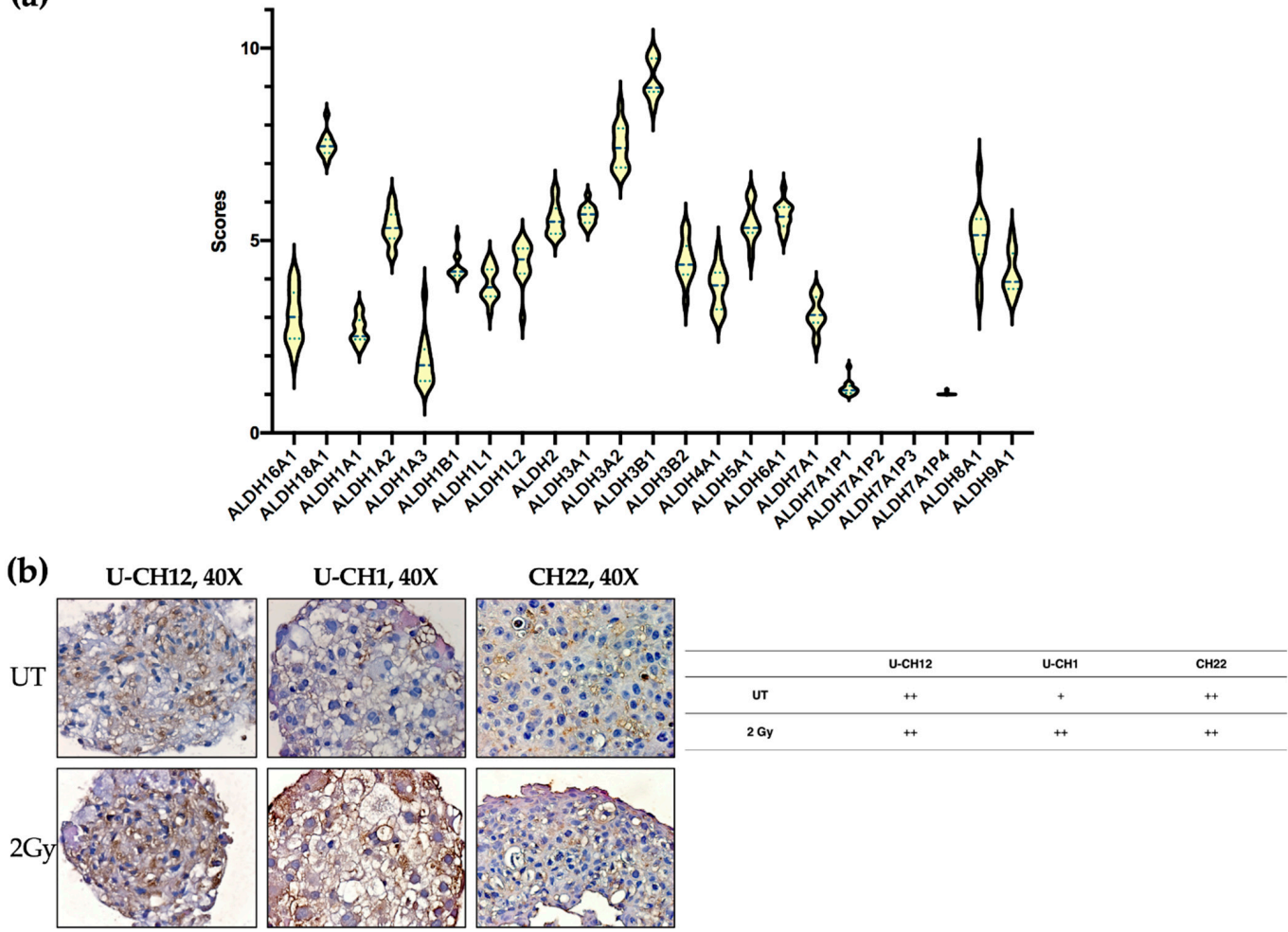

(c)

UT
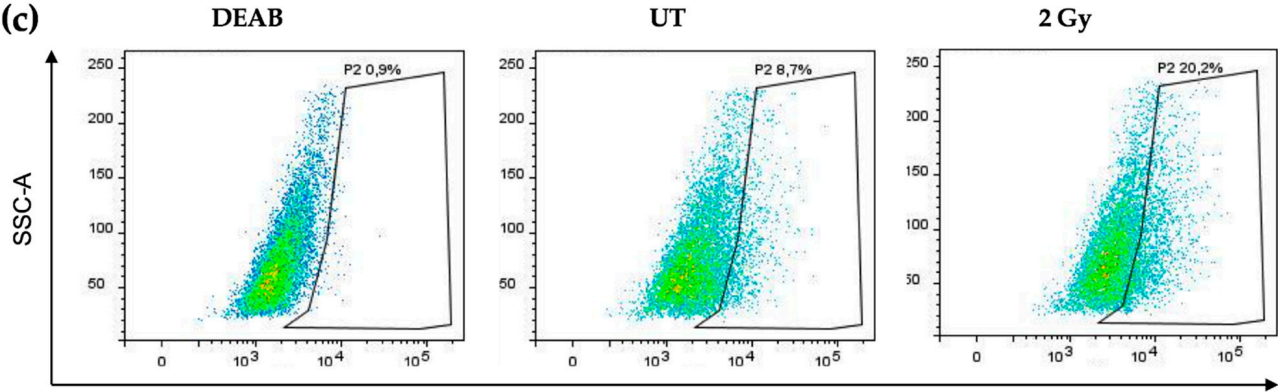

ALDH activity

(d)

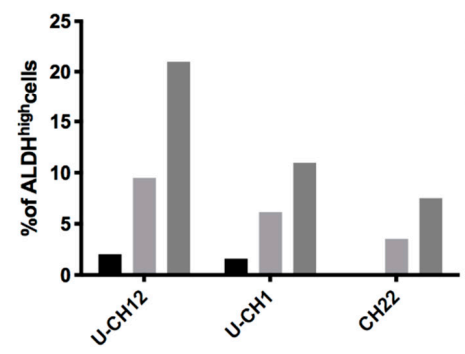

(e)

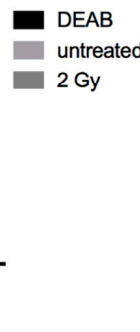

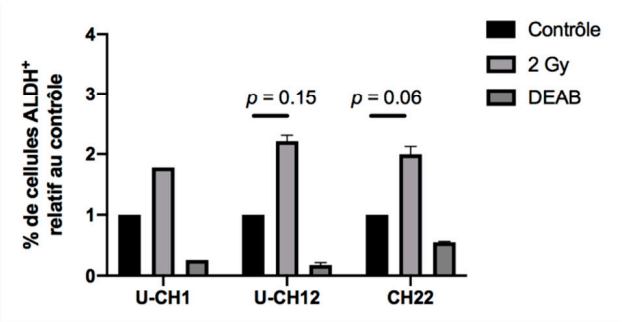

Figure 4. Aldehyde dehydrogenase (ALDH) is a promising radiosensitizing target in chordoma: (a) a graph representing ALDH gene expression scores in 13 chordoma patients, (b) representative images of ALDH3A2 staining after 2 Gy of X-rays or in untreated controls and quantification, (c) images representative of an Aldefluor assay quantifying ALDH1 and 3 activity in response to 2 Gy of X-rays in U-CH12 spheroids, (d) graph representative of the percent of ALDH ${ }^{\text {high }}$ cells, and (e) graph representative of the percent of $\mathrm{ALDH}^{\text {high }}$ cells relative to untreated conditions. Diethylaminobenzaldehyde (DEAB), an inhibitor of ALDH activity, was used as a negative control. The experiment was performed three times in triplicate for U-CH12 and $\mathrm{CH} 22$ spheroids, allowing for a comparison between the UT and 2 Gy groups using 2-way ANOVA. The $p$-value is indicated for each spheroid. 
Next, we focused on the most expressed ALDH enzymes, namely ALDH1 and ALDH3. We investigated whether these enzymes are expressed in chordoma spheroids at the proteomic level. ALDH3 expression was first examined by immunostaining, which revealed that all untreated spheroids moderately expressed ALDH3. This expression increased exclusively in U-CH1 spheroids after radiation (Figure 4). We then evaluated the activity of ALDH1 and ALDH3 in spheroids by flow cytometry using an Aldefluor assay (Figure 4c-e). We used the N,N-diethylaminobenzaldehyde (DEAB) inhibitor of ALDH isoenzymes, as a negative control. These analyses showed that, at the basal level, a small subset of cells present high ALDH activity (U-CH12: 9.5\%; U-CH1: 6.16\%; and $\mathrm{CH} 22: 3.53 \%$ ). This subset of cells augments in response to radiation (U-CH12: $21 \%$; U-CH1: $10.96 \%$; and $\mathrm{CH} 22: 7.5 \%$ ) (Figure 4c), as evidenced by the 2.2-fold increase in $\mathrm{ALDH}^{\text {high }} \mathrm{U}-\mathrm{CH} 12$ cells upon irradiation (U-CH12: 2.2, $p=0.15 ; \mathrm{CH} 22: 1.9, p=0.06$; and U-CH1: 1.8) (Figure 4d). Interestingly, the most radioresistant spheroids, namely U-CH12 spheroids, present the most important population of cells with a high ALDH activity.

Altogether, these results show that aldehyde dehydrogenases are expressed both at the transcriptomic and the proteomic levels in chordoma patients and spheroids. Furthermore, there is an enrichment in cells with ALDH ${ }^{\text {high }}$ activity after radiation. Hence, ALDH1 and ALDH3 may be promising therapeutic targets in chordoma.

\subsection{Inhibiting Aldehyde Dehydrogenase Decreases the Radioresistance of All Three Models}

We hypothesized that the dual inhibition of ALDH1 and ALDH3 could sensitize radioresistant chordoma cells. We first validated that DIMATE was able to specifically inhibit ALDH1 and ALDH3 by flow cytometry using an Aldefluor assay. Both ALDH1 and ALDH3 activity reduced strongly after DIMATE treatment (Figure S2). The IC50 of DIMATE was then established in chordoma spheroids by treating them with 0.1 to $25 \mu \mathrm{M}$ of DIMATE. This IC50 ranged from $1332 \mu \mathrm{M}$ in U-CH1 spheroids to $8836 \mu \mathrm{M}$ in U-CH12 spheroids (Figure 5a). The combined treatment of DIMATE and radiations was applied to spheroids to evaluate their antiproliferative and anti-self-renewal effects. A strong decrease in colony forming ability was observed with the combination treatment at both 10 and $25 \mu \mathrm{M}$, with only 8 and 5 colonies remaining after treatment compared to 37 colonies remaining after radiation alone ( $p<0.0001$ for both concentrations) (Figure $5 \mathrm{~b}, \mathrm{c})$. Moreover, the combination at either 1,10 , or $25 \mu \mathrm{M}$ induced a decrease in colony forming ability more important $(44,8$, and 5 colonies remaining after treatment) than DIMATE alone $(113,78$, and 25 colonies remaining after treatment) at the same concentrations $(p<0.0001$ for all concentrations) (Figure $5 b, c)$. Hence, the combination of DIMATE and radiation triggers a stronger antiproliferative effect. This antiproliferative effect was confirmed in 3D cell cultured conditions by evaluating the capacity of cells to form spheres after the different treatment combinations (Figure $5 \mathrm{~d}, \mathrm{e}$ ). Spheres formed from spheroids treated with the combination at $25 \mu \mathrm{M}$ displayed a 3 times smaller area ( $2 \mathrm{~Gy}, \mathrm{D} 25 \mu \mathrm{M}=0.33$ ) compared to the untreated control (UT $=1 ; p<0.0001$ ) (Figure $5 \mathrm{~d}$,e). The capacity to form spheres decreased and the newly formed spheres progressed less rapidly when spheres were formed following the combination treatment compared to those formed from radiation alone, indicative of a strong cytostatic effect. 
(a)

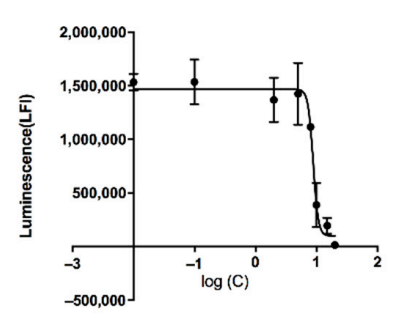

(b)

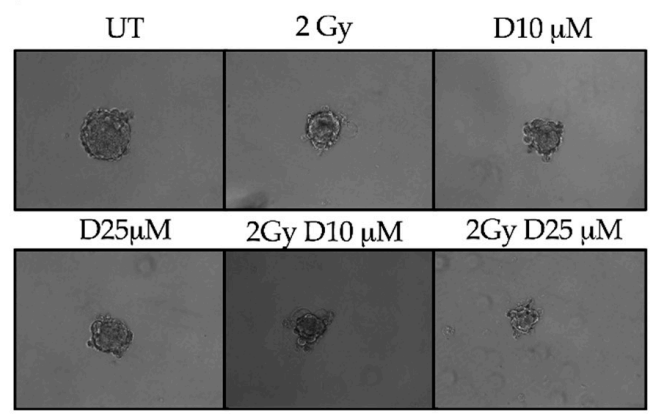

U-CH1

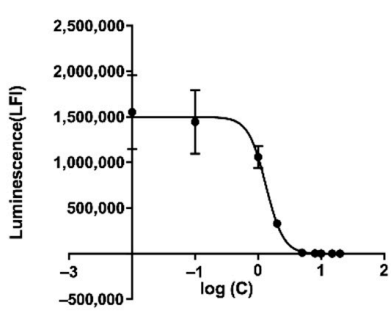

$\mathrm{IC} 50=1.332 \mu \mathrm{M}$
$\mathrm{CH} 22$

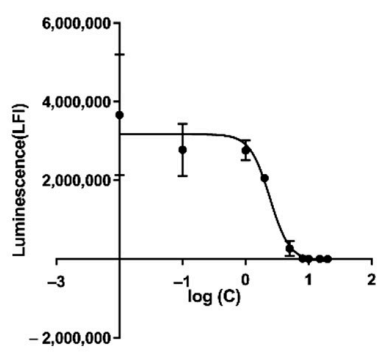

IC50 $=2.425 \mu \mathrm{M}$

(c)

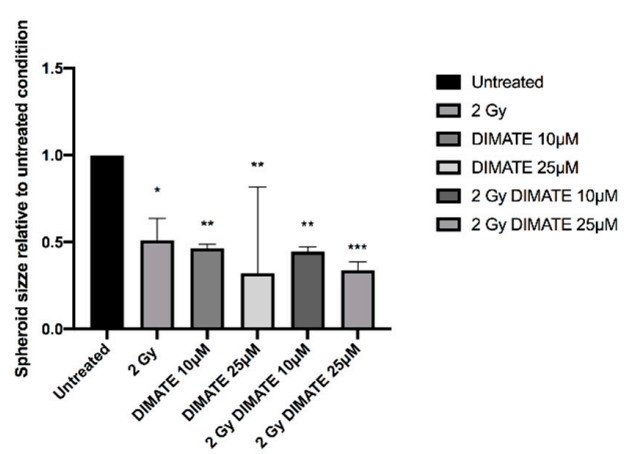

(d)

(e)
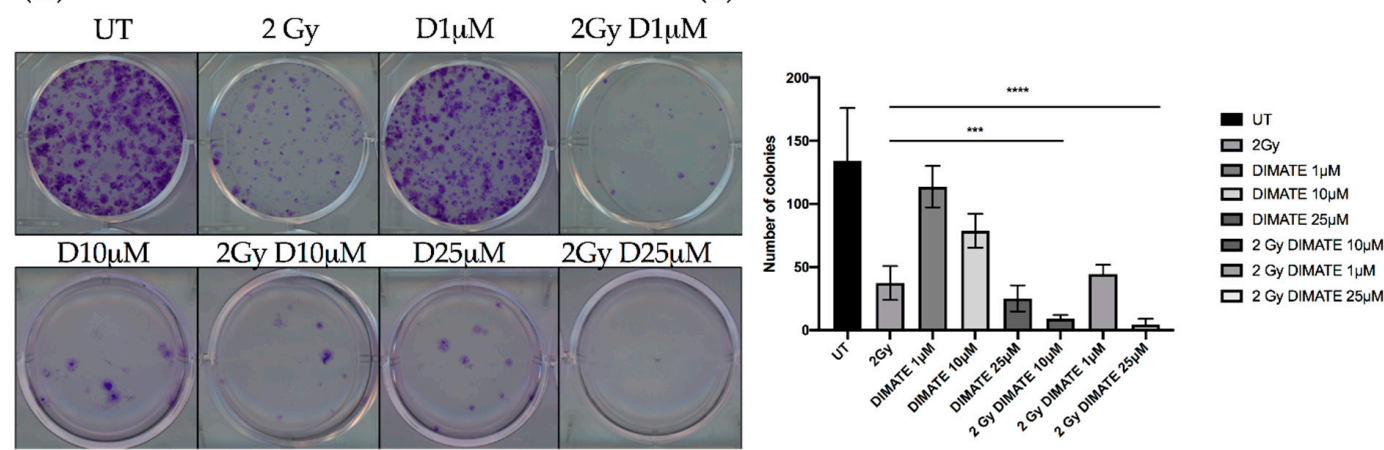

(f)

$\mathrm{U}-\mathrm{CH} 12$

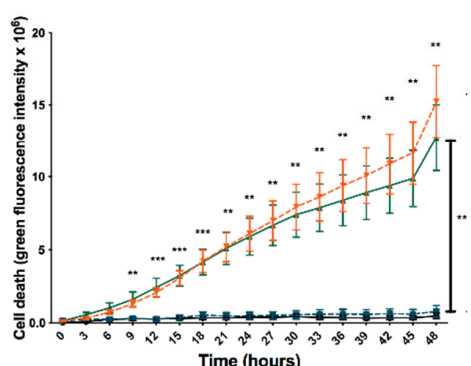

Time (hours)
U-CH1

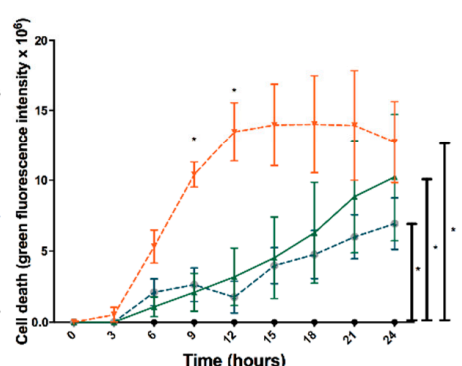

Time (hours)
$\mathrm{CH} 22$

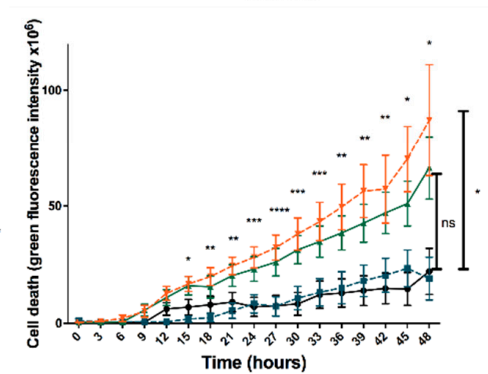

Time (hours)

$$
\text { Control } \rightarrow 2 \text { Gy } \quad \rightarrow \text { DIMATE } 25 \mu \mathrm{M} \quad-\approx-2 \text { Gy + DIMATE } 25 \mu \mathrm{M}
$$

Figure 5. ALDH inhibition decreases radioresistance in chordoma: (a) a graph representative of three independent experiments of DIMATE IC50 in chordoma spheroids after $48 \mathrm{~h}$ of treatment at concentrations ranging from 0.1 to $25 \mu \mathrm{M}$, (b) images and (c) a graph representative of the $\mathrm{CH} 22$ spheroid size relative to untreated conditions after treatment with X-rays or after a combined treatment with X-rays and DIMATE, (d) images and (e) a graph representative of the number of colonies formed in UT conditions or after treatment with DIMATE as a monotherapy or combined with 2 Gy of X-rays in CH22 spheroids, and (f) a graph representative of spheroid cell death over $48 \mathrm{~h}$ in UT conditions or after treatment with DIMATE alone or in combination with 2 Gy of X-rays. Each experiment was conducted three times in triplicate. The statistical comparison between each group was determined using a one-way ANOVA. Significant $p$-values are indicated as follows: $p<0.05^{*}, p<0.01^{* *}, p<0.001^{* * *}$, and $p<0.0001^{* * * *}$. 
We then evaluated whether DIMATE, radiation, or their combination induced cell death. Spheroids treated with DIMATE alone or in combination with radiation showed a strong induction of cell death after 24 or $48 \mathrm{~h}$ of treatment (U-CH12: $p<0.01$; U-CH1: $p<0.05$; and CH22: $p<0.05$ ). No significant difference in the induction of cell death was observed between spheroids treated with DIMATE alone or with the combination of DIMATE and radiotherapy for U-CH12 and $\mathrm{CH} 22$. However, the treatment combination was more efficient at inducing cell death in U-CH1 spheroids, with a faster induction of cell death $(9 \mathrm{~h}$ and $12 \mathrm{~h}$ of treatment, $p<0.05)$. Moreover, the treatment combination was more effective than radiation alone in all 3 spheroids, $9 \mathrm{~h}$ after treatment onwards for U-CH12 and $\mathrm{U}-\mathrm{CH} 1$ spheroids ( $p<0.01$ and $p<0.05$, respectively), and after $15 \mathrm{~h}$ of treatment for $\mathrm{CH} 22(p<0.05)$ (Figure 5f). Hence, DIMATE exhibited not only cytostatic effects but also cytotoxic effects able to reinduce cell death in combination with radiation. Hence, the inhibition of ALDH1 and ALDH3 reinduced radiosensitivity in chordoma spheroids.

\section{Discussion}

Chordoma patients currently rely on aggressive surgery and high-dose radiotherapy. The infrequency of chordoma has so far delayed and obstructed the development of efficient targeted therapies against these tumors. It has been demonstrated that $95 \%$ of the drugs in phase I of clinical trials never obtain FDA approval [30]. In the last decade, efforts have been made to understand this lack of efficacy, which may partly be due to the low predictive value of preclinical models. Indeed, only a few cell lines and patient-derived xenografts (PDX) are available as in vitro and in vivo models of chordoma. To better predict response to drugs, we thus needed to create models at the crossroad between cell lines and PDXs. In this study, we established, characterized, and used 3D cellular models of chordoma for the preclinical evaluation of a radiosensitizing strategy. First, these models are representative of the histological and immunohistochemical features of the disease. Interestingly, brachyury had higher expression levels in $\mathrm{CH} 22$ spheroids than in U-CH12 spheroids. Indeed, brachyury overexpression has been detected in several epithelial cancers and was shown to promote the epithelial-to-mesenchymal transition that enables tumors to metastasize. The levels of brachyury expression have been correlated with disease stage, poor prognosis, and tumor resistance to cytotoxic therapies in a number of cancers [31-34]. In chordoma, brachyury overexpression is also associated with shorter progression-free survival [35]. Thus, the difference in expression of brachyury between primary and metastatic spheroids could be due to the role of brachyury in tumor progression. These models also recapitulate the peculiar radioresistant environment of chordoma and the progression of the disease (primary, relapse, and metastatic).

The current limitations of cell culture are now partially overcome by $3 \mathrm{D}$ cell cultures. Three-dimensional cell cultures better mimic the native cancer tissue, since they restore some of the specific biochemical and morphological features seen in vivo [36,37]. In our models, important morphological features were observed with the presence of the extracellular matrix in U-CH12 and U-CH1 composed of proteoglycans and collagens. Moreover, while cells are equally exposed to drugs in 2D cultures, in chordoma 3D models, we recapitulated the difficulty of drugs to gain access to tumor cells. Over the last few years, new complex 3D models have emerged, with the most interesting one remaining organoids. Organoids allow us to collect the primary culture of samples from patients and to maintain intra-tumoral heterogeneity [38]. One study has so far reported the establishment of organoid models of chordomas in a preclinical context to predict the response to PD-1/PDL1 checkpoint inhibitors [39]. Despite these promising results, a deeper characterization of these primary cell cultures is needed. Chordoma spheroids contain different types of cells: proliferating, quiescent, hypoxic, and mimicking the diversity found within a tumor cell. Moreover, in vivo models of chordoma are interesting because they are able to progress. However, owing to the scarcity of chordoma samples and the slow growth rate of these tumors, organoid development has been hindered. Here, we addressed tumor progression by using cell lines established from a primary, a relapsed, and a metastatic tumor from 
different patients. Finally, our models match the clinical description of chordoma: primary tumors have low cellularity, slow growth, and an abundant extracellular matrix, while metastatic tumors have high cellularity, faster growth, and less extracellular matrixes.

Preclinical in vivo models are also of utmost importance to determine the bioavailability of drugs in a living organism. Giving the differences between our 3D models, it was not surprising to obtain variable levels of radioresistance, reflecting what is observed in the clinic [6]. As patients with sacral chordomas are radioresistant, they are treated with fractionated high-dose radiotherapy with a maximal dose of $2 \mathrm{~Gy} /$ per session [6]. We also subjected our models to this dose to mimic clinical settings. To our knowledge, only two studies have so far focused on chordoma radioresistance. The first study demonstrated that U-CH1 cells grown in 2D have a normal radiosensitivity range, whereas we found a strong radioresistance [40]. This difference may be due to an alteration in the cell line used, as the authors kept only one highly dividing subclone. We thus speculate that the quiescent and low dividing subclones must be at the origin of radioresistance in this cell line. Confirming this hypothesis, the second study evaluated the response of the U-CH1 cell line to ionizing radiation and found the same rate of survival at $2 \mathrm{~Gy}$ of $\mathrm{X}$-rays as ours [41]. The variable degrees of radioresistance found can also be explained by the differences in proliferation, composition of extracellular matrix, and hypoxia. The extracellular matrix is known to increase cell adhesion and drives radioresistance. Moreover, hypoxia is a cause of treatment failure, and new radiotherapeutic strategies aim at delivering a stronger dose to hypoxic tumor zones to eliminate chordoma tumors [13,42]. In the same intent of improving chordoma patient response to radiotherapy, proton irradiations are used in chordomas in recent clinical trials and show better results than X-rays [43,44]. Even though proton therapy is still poorly accessible, it could be interesting to study the response of our spheroids to this kind of treatment. The chordoma models we established present 3 different levels of radioresistance; hence, such models can be very helpful to evaluate radiosensitizing therapies and to study and compare their effects on low or highly radioresistant cells.

We thus also tested a radiosensitizing approach based on ALDH targeting. Aside from their role in protection against oxidative stress as detoxifying enzymes [15], ALDHs are involved in stem cell maintenance and are the principal marker of cancer stem cells [45]. ALDH1 and ALDH3 are the most extensively studied ALDHs in cancer. Cancer stem cells have increased activity of ALDH1 and ALDH3, and this is particularly used to isolate them from tumor bulk. They are involved in resistance to both chemo- and radiotherapy in most solid tumors [16-18,21,46]. In chordoma, the ALDH activity has been measured in cell lines. These studies demonstrated that a population of U-CH1 cells (between 0.48 and $2.5 \%$ depending on the study) have a high ALDH activity $[23,24]$. In comparison, we found a more important basal rate of ALDH ${ }^{\text {high }}$ cells (5\% in UT conditions). This can be explained by the fact that we grew the cells in 3D. Indeed, in another study, the JHC7 cell line presented an increase in the ALDH ${ }^{\text {high }}$ population when cultured in 3D in comparison to adherent cells cultures [25]. In those studies, the ALDH activity was used to isolate cancer stem cells, and this activity was correlated with increased tumor initiation capacities and with the expression of genes involved in stem cell maintenance. Cancer stem cells have also been detected in chordoma patients and cell lines (JHC7, U-CH1, and U-CH2) [23-25,47]. Here, we confirmed the presence of a subpopulation of cells with a high ALDH activity in 3 different models, but we also correlated this activity with resistance to radiation. This subpopulation of cells could potentially be stem cells.

Finally, we tested the efficacy of an inhibitor of ALDH1/3 (DIMATE). DIMATE has well-known antiproliferative effects in acute myleoid leukemia, non-small cell lung cancer (NSCLC), melanoma, and prostate cancer in vitro [26,48-50]. This inhibitor has been used as a monotherapy or combined with a chemotherapeutic agent inducing ROS production in NSCLC, with the aim of disrupting ROS balance in cells to induce death. The combination of DIMATE and CDDP in NSCLC has a synergistic effect on cell death in vitro and in vivo [26]. Here, we combined DIMATE with radiotherapy (X-rays). An important part of the effects produced by radiotherapy rely on ROS, which enhance DNA damage and lead to cell death. 
Our results show that the combination of DIMATE and radiation induces a strong inhibition of self-renewal capacities with complete inhibition of both the colony forming and the sphere forming abilities. Moreover, an important induction of cell death was observed with DIMATE both in mono- and combined therapy. The induction was accelerated in $\mathrm{U}-\mathrm{CH} 1$ spheroids when the treatments were combined. Such an effect has never been obtained in vitro in chordoma, making ALDH a promising target for future preclinical experiments. Interestingly, the inhibition of RAD51 has been shown to sensitize chordoma cells to radiations in vitro [41]. Moreover, this inhibition also sensitizes cells to aldehyde treatment, substrates of ALDH activity [51]. As our work shows that the inhibition of ALDH activity sensitizes cells to radiations, we wonder whether the inhibition of both RAD51 and ALDH could have synergistic effects and improved response to radiation in chordoma. In conclusion, we created 3D cellular models of sacral chordoma derived from primary, recurrent, and metastatic tumors that reproduce histological and therapeutic features of the disease. These models can be used to decrypt chordoma alterations, in mechanisms of progression, and for preclinical evaluation of drugs. We show for the first time the benefits of using dual inhibitors of ALDH1 and 3 as radiosensitizing agents in chordoma in vitro and provide preclinical support for their use as monotherapies or in combination with radiotherapy in chordoma.

Supplementary Materials: The following are available online at https://www.mdpi.com/2073-4 409/10/2/399/s1, Figure S1: Comparison of RNA sequencing data from spheroids and chordoma patient samples; Figure S2: Graph representative of an aldefluor assay using flow cytometry after treatment with DIMATE; Table S1: Table representing the most up-regulated genes between chordoma spheroids and patient samples in the differentially expressed genes analysis; Table S2: Table representing the most down-regulated genes between chordoma spheroids and patient samples in the differentially expressed genes analysis.

Author Contributions: M.-A.L. conduced the experiments, acquired data, analyzed the data, wrote and revised the manuscript. A.-L.D., P.B., L.A., I.R., J.B., J.V. acquired data. D.P. and F.T. acquired and analyzed RNA sequencing data. M.K., R.P., O.G. constituted the patient cohort and their clinical information. A.D. and J.-Y.B. designed the research study and A.D. wrote the manuscript. All authors have read and agreed to the published version of the manuscript.

Funding: This research received no external funding.

Institutional Review Board Statement: The study was conducted according to the guidelines of the Declaration of Helsinki and approved by the Clinical Research Ethics Committee of the Centre Leon Berard, ethical approval number 2018-014, approval date 3 October 2018.

Informed Consent Statement: Informed consent was obtained from all subjects involved in the study.

Data Availability Statement: The data presented in this study are available on request from the corresponding author. The data are not publicly available because chordoma cohort is being expanding and more molecular analysis are ongoing and planed.

Acknowledgments: The authors acknowledge Advanced BioDesign (Bron, France) for the DIMATE furniture and the Chordoma Foundation for providing the cell lines.

Conflicts of Interest: The authors declare no conflict of interest.

\section{References}

1. Das, P.; Soni, P.; Jones, J.; Habboub, G.; Barnholtz-Sloan, J.S.; Recinos, P.F.; Kshettry, V.R. Descriptive Epidemiology of Chordomas in the United States. J. Neurooncol. 2020, 148, 173-178. [CrossRef] [PubMed]

2. Smoll, N.R.; Gautschi, O.P.; Radovanovic, I.; Schaller, K.; Weber, D.C. Incidence and Relative Survival of Chordomas: The Standardized Mortality Ratio and the Impact of Chordomas on a Population. Cancer 2013, 119, 2029-2037. [CrossRef] [PubMed]

3. Gatta, G.; Capocaccia, R.; Botta, L.; Mallone, S.; Angelis, R.D.; Ardanaz, E.; Comber, H.; Dimitrova, N.; Leinonen, M.K.; Siesling, S.; et al. Burden and Centralised Treatment in Europe of Rare Tumours: Results of RARECAREnet—a Population-Based Study. Lancet Oncol. 2017, 18, 1022-1039. [CrossRef]

4. Radaelli, S.; Fossati, P.; Stacchiotti, S.; Akiyama, T.; Asencio, J.M.; Bandiera, S.; Boglione, A.; Boland, P.; Bolle, S.; Bruland, Ø.; et al. The Sacral Chordoma Margin. Eur. J. Med. Chem. 2020. [CrossRef] 
5. Uhrenholt, L.; Stimpel, H. Histochemistry of Sacrococcygeal Chordoma. Acta Pathol. Microbiol. Immunol. Scand. A. 1985, 93A, 203-204. [CrossRef]

6. Heery, C.R. Chordoma: The Quest for Better Treatment Options. Oncol. Ther. 2016, 4, 35-51. [CrossRef]

7. Stacchiotti, S.; Sommer, J. Chordoma Global Consensus Group Building a Global Consensus Approach to Chordoma: A Position Paper from the Medical and Patient Community. Lancet Oncol. 2015, 16, e71-e83. [CrossRef]

8. Stacchiotti, S.; Gronchi, A.; Fossati, P.; Akiyama, T.; Alapetite, C.; Baumann, M.; Blay, J.Y.; Bolle, S.; Boriani, S.; Bruzzi, P.; et al. Best Practices for the Management of Local-Regional Recurrent Chordoma: A Position Paper by the Chordoma Global Consensus Group. Available online: https:/ / pubmed.ncbi.nlm.nih.gov/28184416/ (accessed on 20 May 2020).

9. Holliday, E.B.; Mitra, H.S.; Somerson, J.S.; Rhines, L.D.; Mahajan, A.; Brown, P.D.; Grosshans, D.R. Postoperative Proton Therapy for Chordomas and Chondrosarcomas of the Spine: Adjuvant versus Salvage Radiation Therapy. Spine 2015, 40, 544-549. [CrossRef]

10. Tarpey, P.S.; Behjati, S.; Young, M.D.; Martincorena, I.; Alexandrov, L.B.; Farndon, S.J.; Guzzo, C.; Hardy, C.; Latimer, C.; Butler, A.P.; et al. The Driver Landscape of Sporadic Chordoma. Nat. Commun. 2017, 8, 890. [CrossRef]

11. Frezza, A.M.; Botta, L.; Trama, A.; Dei Tos, A.P.; Stacchiotti, S. Chordoma: Update on Disease, Epidemiology, Biology and Medical Therapies. Curr. Opin. Oncol. 2019, 31, 114-120. [CrossRef]

12. Cheney, M.D.; Chen, Y.-L.; Lim, R.; Winrich, B.K.; Grosu, A.L.; Trofimov, A.V.; Depauw, N.; Shih, H.A.; Schwab, J.H.; Hornicek, F.J.; et al. 18F-FMISO PET/CT Visualization of Tumor Hypoxia in Patients with Chordoma of the Mobile and Sacrococcygeal Spine. Int. J. Radiat. Oncol. Biol. Phys. 2014, 90, 1030-1036. [CrossRef]

13. Chen, Y.L.E.; Faridi, K.; Wang, H.; Lim, R.; Schroeder, H.; Bernstein, K.; Choy, E.; Hornicek, F.J.; DeLaney, T.F. Predictive Value of FMISO Positron Emission Tomography/Computed Tomography Hypoxic Subvolume for Long-Term Disease Recurrence or Metastases in Mobile Spine and Sacrococcygeal Chordoma. Int. J. Radiat. Oncol. Biol. Phys. 2016, 96, S216-S217. [CrossRef]

14. Jensen, C.; Teng, Y. Is It Time to Start Transitioning From 2D to 3D Cell Culture? Front. Mol. Biosci. 2020, 7, 33. [CrossRef] [PubMed]

15. Singh, S.; Brocker, C.; Koppaka, V.; Ying, C.; Jackson, B.; Matsumoto, A.; Thompson, D.C.; Vasiliou, V. Aldehyde Dehydrogenases in Cellular Responses to Oxidative/Electrophilic Stress. Free Radic. Biol. Med. 2013, 56, 89-101. [CrossRef]

16. Dehghan Harati, M.; Rodemann, H.P.; Toulany, M. Nanog Signaling Mediates Radioresistance in ALDH-Positive Breast Cancer Cells. Int. J. Mol. Sci. 2019, 20. [CrossRef] [PubMed]

17. Croker, A.K.; Allan, A.L. Inhibition of Aldehyde Dehydrogenase (ALDH) Activity Reduces Chemotherapy and Radiation Resistance of Stem-like ALDHhiCD44+ Human Breast Cancer Cells. Breast Cancer Res. Treat 2012, 133, 75-87. [CrossRef] [PubMed]

18. Yao, T.; Weng, X.; Yao, Y.; Huang, C.; Li, J.; Peng, Y.; Lin, R.; Lin, Z. ALDH-1-Positive Cells Exhibited a Radioresistant Phenotype That Was Enhanced with Hypoxia in Cervical Cancer. BMC Cancer 2020, 20, 891. [CrossRef] [PubMed]

19. Cojoc, M.; Peitzsch, C.; Kurth, I.; Trautmann, F.; Kunz-Schughart, L.A.; Telegeev, G.D.; Stakhovsky, E.A.; Walker, J.R.; Simin, K.; Lyle, S.; et al. Aldehyde Dehydrogenase Is Regulated by $\beta$-Catenin/TCF and Promotes Radioresistance in Prostate Cancer Progenitor Cells. Cancer Res. 2015, 75, 1482-1494. [CrossRef]

20. Kurth, I.; Hein, L.; Mäbert, K.; Peitzsch, C.; Koi, L.; Cojoc, M.; Kunz-Schughart, L.; Baumann, M.; Dubrovska, A. Cancer Stem Cell Related Markers of Radioresistance in Head and Neck Squamous Cell Carcinoma. Oncotarget 2015, 6, 34494-34509. [CrossRef]

21. Awad, O.; Yustein, J.T.; Shah, P.; Gul, N.; Katuri, V.; O’Neill, A.; Kong, Y.; Brown, M.L.; Toretsky, J.A.; Loeb, D.M. High ALDH Activity Identifies Chemotherapy-Resistant Ewing's Sarcoma Stem Cells That Retain Sensitivity to EWS-FLI1 Inhibition. PLoS ONE 2010, 5, e13943. [CrossRef] [PubMed]

22. Aydemir, E.; Bayrak, O.F.; Sahin, F.; Atalay, B.; Kose, G.T.; Ozen, M.; Sevli, S.; Dalan, A.B.; Yalvac, M.E.; Dogruluk, T.; et al. Characterization of Cancer Stem-like Cells in Chordoma: Laboratory Investigation. J. Neurosurg. 2012, 116, 810-820. [CrossRef] [PubMed]

23. Tuysuz, E.C.; Gulluoglu, S.; Yaltirik, C.K.; Ozbey, U.; Kuskucu, A.; Çoban, E.A.; Sahin, F.; Türe, U.; Bayrak, O.F. Distinctive Role of Dysregulated MiRNAs in Chordoma Cancer Stem-like Cell Maintenance. Exp. Cell Res. 2019, 380, 9-19. [CrossRef]

24. Owen, J.H.; Komarck, C.M.; Wang, A.C.; Abuzeid, W.M.; Keep, R.F.; McKean, E.L.; Sullivan, S.; Fan, X.; Prince, M.E.P. UM-Chor1: Establishment and Characterization of the First Validated Clival Chordoma Cell Line. J. Neurosurg. 2017, 128, 701-709. [CrossRef]

25. Shah, S.R.; David, J.M.; Tippens, N.D.; Mohyeldin, A.; Martinez-Gutierrez, J.C.; Ganaha, S.; Schiapparelli, P.; Hamilton, D.H.; Palena, C.; Levchenko, A.; et al. Brachyury-YAP Regulatory Axis Drives Stemness and Growth in Cancer. Cell Rep. 2017, 21, 495-507. [CrossRef] [PubMed]

26. Rebollido-Rios, R.; Venton, G.; Sánchez-Redondo, S.; Felip, C.I.; Fournet, G.; González, E.; Fernández, W.R.; Escuela, D.O.B.; Stefano, B.D.; Penarroche-Díaz, R.; et al. Dual Disruption of Aldehyde Dehydrogenases 1 and 3 Promotes Functional Changes in the Glutathione Redox System and Enhances Chemosensitivity in Nonsmall Cell Lung Cancer. Oncogene 2020, 39, $2756-2771$. [CrossRef] [PubMed]

27. Dinavahi, S.S.; Gowda, R.; Bazewicz, C.G.; Battu, M.B.; Lin, J.M.; Chitren, R.J.; Pandey, M.K.; Amin, S.; Robertson, G.P.; Gowda, K. Design, Synthesis Characterization and Biological Evaluation of Novel Multi-Isoform ALDH Inhibitors as Potential Anticancer Agents. Eur. J. Med. Chem. 2020, 187, 111962. [CrossRef] [PubMed] 
28. Koppaka, V.; Thompson, D.C.; Chen, Y.; Ellermann, M.; Nicolaou, K.C.; Juvonen, R.O.; Petersen, D.; Deitrich, R.A.; Hurley, T.D.; Vasiliou, V. Aldehyde Dehydrogenase Inhibitors: A Comprehensive Review of the Pharmacology, Mechanism of Action, Substrate Specificity, and Clinical Application. Pharmacol. Rev. 2012, 64, 520-539. [CrossRef] [PubMed]

29. van der Maaten, L.; Hinton, G. Visualizing Data Using T-SNE. J. Mach. Learn. Res. 2008, 9, 2579-2605.

30. Moreno, L.; Pearson, A.D.J. How Can Attrition Rates Be Reduced in Cancer Drug Discovery? Expert. Opin. Drug Discov. 2013, 8, 363-368. [CrossRef]

31. Roselli, M.; Fernando, R.I.; Guadagni, F.; Spila, A.; Alessandroni, J.; Palmirotta, R.; Costarelli, L.; Litzinger, M.; Hamilton, D.; Huang, B.; et al. Brachyury, a Driver of the Epithelial-Mesenchymal Transition, Is Overexpressed in Human Lung Tumors: An Opportunity for Novel Interventions against Lung Cancer. Clin. Cancer Res. 2012, 18, 3868-3879. [CrossRef]

32. Wan, Z.; Jiang, D.; Chen, S.; Jiao, J.; Ji, L.; Shah, A.S.; Wei, H.; Yang, X.; Li, X.; Wang, Y.; et al. T-Box Transcription Factor Brachyury Promotes Tumor Cell Invasion and Metastasis in Non-Small Cell Lung Cancer via Upregulation of Matrix Metalloproteinase 12. Oncol. Rep. 2016, 36, 306-314. [CrossRef] [PubMed]

33. Chen, M.; Zou, S.; He, C.; Zhou, J.; Li, S.; Shen, M.; Cheng, R.; Wang, D.; Zou, T.; Yan, X.; et al. Transactivation of SOX5 by Brachyury Promotes Breast Cancer Bone Metastasis. Carcinogenesis 2020, 41, 551-560. [CrossRef]

34. Du, R.; Wu, S.; Lv, X.; Fang, H.; Wu, S.; Kang, J. Overexpression of Brachyury Contributes to Tumor Metastasis by Inducing Epithelial-Mesenchymal Transition in Hepatocellular Carcinoma. J. Exp. Clin. Cancer Res. 2014, 33, 105. [CrossRef] [PubMed]

35. Otani, R.; Mukasa, A.; Shin, M.; Omata, M.; Takayanagi, S.; Tanaka, S.; Ueki, K.; Saito, N. Brachyury Gene Copy Number Gain and Activation of the PI3K/Akt Pathway: Association with Upregulation of Oncogenic Brachyury Expression in Skull Base Chordoma. J. Neurosurg. 2018, 128, 1428-1437. [CrossRef] [PubMed]

36. Fang, Y.; Eglen, R.M. Three-Dimensional Cell Cultures in Drug Discovery and Development. SLAS Discov. 2017, $22,456-472$. [CrossRef] [PubMed]

37. Langhans, S.A. Three-Dimensional in Vitro Cell Culture Models in Drug Discovery and Drug Repositioning. Front Pharmacol. 2018, 9, 6. [CrossRef]

38. Garreta, E.; Kamm, R.D.; Chuva de Sousa Lopes, S.M.; Lancaster, M.A.; Weiss, R.; Trepat, X.; Hyun, I.; Montserrat, N. Rethinking Organoid Technology through Bioengineering. Nat. Mater 2020. [CrossRef] [PubMed]

39. Scognamiglio, G.; De Chiara, A.; Parafioriti, A.; Armiraglio, E.; Fazioli, F.; Gallo, M.; Aversa, L.; Camerlingo, R.; Cacciatore, F.; Colella, G.; et al. Patient-Derived Organoids as a Potential Model to Predict Response to PD-1/PD-L1 Checkpoint Inhibitors. Br. J. Cancer. 2019, 121, 979-982. [CrossRef]

40. Kato, T.A.; Tsuda, A.; Uesaka, M.; Fujimori, A.; Kamada, T.; Tsujii, H.; Okayasu, R. In Vitro Characterization of Cells Derived from Chordoma Cell Line U-CH1 Following Treatment with X-Rays, Heavy Ions and Chemotherapeutic Drugs. Radiat. Oncol. 2011, 6, 116. [CrossRef]

41. Zhang, C.; Wang, B.; Li, L.; Li, Y.; Li, P.; Lv, G. Radioresistance of Chordoma Cells Is Associated with the ATM/ATR Pathway, in Which RAD51 Serves as an Important Downstream Effector. Exp. Ther. Med. 2017, 14, 2171-2179. [CrossRef]

42. Yan, S.; Broussard, G.; De, K.; Bernstein, A.; Wang, Y. SU-F-T-203: High Dose Definitive Proton Therapy with Integrated Boost to Hypoxic Subvolumes Determined by [18F]-Fluoromisonidazole (FMISO) PET/CT for Patients with Recurrent Chordomas Using Intensity Modulated Proton Therapy (IMPT). Med. Phys. 2016, 43, 3508-3509. [CrossRef]

43. Aibe, N.; Demizu, Y.; Sulaiman, N.S.; Matsuo, Y.; Mima, M.; Nagano, F.; Terashima, K.; Tokumaru, S.; Hayakawa, T.; Suga, M.; et al. Outcomes of Patients With Primary Sacral Chordoma Treated With Definitive Proton Beam Therapy. Int. J. Radiat. Oncol. Biol. Phys. 2018, 100, 972-979. [CrossRef] [PubMed]

44. Baumann, B.C.; Lustig, R.A.; Mazzoni, S.; Grady, S.M.; O’Malley, B.W.; Lee, J.Y.K.; Newman, J.G.; Schuster, J.M.; Both, S.; Lin, A.; et al. A Prospective Clinical Trial of Proton Therapy for Chordoma and Chondrosarcoma: Feasibility Assessment. J. Surg. Oncol. 2019, 120, 200-205. [CrossRef]

45. Clark, D.W.; Palle, K. Aldehyde Dehydrogenases in Cancer Stem Cells: Potential as Therapeutic Targets. Ann. Transl. Med. 2016, 4. [CrossRef] [PubMed]

46. Bidan, N.; Bailleul-Dubois, J.; Duval, J.; Winter, M.; Denoulet, M.; Hannebicque, K.; El-Sayed, I.Y.; Ginestier, C.; Forissier, V.; Charafe-Jauffret, E.; et al. Transcriptomic Analysis of Breast Cancer Stem Cells and Development of a PALDH1A1:MNeptune Reporter System for Live Tracking. Proteomics 2019, 19, e1800454. [CrossRef] [PubMed]

47. Fujii, R.; Friedman, E.R.; Richards, J.; Tsang, K.Y.; Heery, C.R.; Schlom, J.; Hodge, J.W. Enhanced Killing of Chordoma Cells by Antibody-Dependent Cell-Mediated Cytotoxicity Employing the Novel Anti-PD-L1 Antibody Avelumab. Oncotarget 2016, 7, 33498-33511. [CrossRef] [PubMed]

48. Venton, G.; Pérez-Alea, M.; Baier, C.; Fournet, G.; Quash, G.; Labiad, Y.; Martin, G.; Sanderson, F.; Poullin, P.; Suchon, P.; et al. Aldehyde Dehydrogenases Inhibition Eradicates Leukemia Stem Cells While Sparing Normal Progenitors. Blood Cancer J 2016, 6, e469. [CrossRef]

49. Quash, G.; Fournet, G.; Courvoisier, C.; Martinez, R.M.; Chantepie, J.; Paret, M.J.; Pharaboz, J.; Joly-Pharaboz, M.O.; Goré, J.; André, J.; et al. Aldehyde Dehydrogenase Inhibitors: $\alpha, \beta$-Acetylenic N-Substituted Aminothiolesters Are Reversible Growth Inhibitors of Normal Epithelial but Irreversible Apoptogens for Cancer Epithelial Cells from Human Prostate in Culture. Eur. J. Med. Chem. 2008, 43, 906-916. [CrossRef] 
50. Pérez-Alea, M.; McGrail, K.; Sánchez-Redondo, S.; Ferrer, B.; Fournet, G.; Cortés, J.; Muñoz, E.; Hernandez-Losa, J.; Tenbaum, S.; Martin, G.; et al. ALDH1A3 Is Epigenetically Regulated during Melanocyte Transformation and Is a Target for Melanoma Treatment. Oncogene 2017, 36, 5695-5708. [CrossRef]

51. Tacconi, E.M.; Lai, X.; Folio, C.; Porru, M.; Zonderland, G.; Badie, S.; Michl, J.; Sechi, I.; Rogier, M.; Matía García, V.; et al. BRCA1 and BRCA2 Tumor Suppressors Protect against Endogenous Acetaldehyde Toxicity. EMBO Mol. Med. 2017, 9, $1398-1414$. [CrossRef] 